\title{
Continious Cultivation of Mixotrophic Microalgae in Membrane Bioreactor for Ammonium Removal
}

\author{
Azadeh Babaei ${ }^{1}$ and Jalal Shayegan ${ }^{2 *}$ \\ ${ }^{1}$ School of Chemical Engineering, University of Tehran, Iran \\ ${ }^{2}$ Department of Chemical and Petroleum Engineering, Sharif University of Technology, Iran
}

*Corresponding author: Jalal Shayegan, Department of Chemical and Petroleum Engineering, Sharif University of Technology, Tehran, Iran , Tel: +982166165420; Fax: +98 2166022853; Email: shayegan@sharif.edu

Submission: 㭰June 11, 2018; Published: 眥 June 14, 2018

\begin{abstract}
This experimental protocol was defined to examine the effect of mixotrophic culture of Chlorella vulgaris on the ammonia removal and microalgae biomass in batch and continuous cultivation. The maximum microalgae concentration was achieved on day 23 (1.3gr/L), respectively. The microalgae were able to reduce the $\mathrm{N}-\mathrm{NH}_{3}$ concentration to $80 \%$ in continuous mode.
\end{abstract}

Keywords: Ammonium removal; Mixotrophic culture; Membrane bioreactor

\section{Introduction}

Microalgae are used to wastewater treatment and produce biofuel [1]. Under mixotrophic conditions algae can uptake inorganic and organic carbon for photosynthesis and other metabolic pathways. Results of several studies have shown that higher biomass production of algae can be achieved by cultivating algae under heterotrophic and mixotrophic conditions, compared to the autotrophic mode of growth $[2,3]$.

Innovative wastewater treatment technologies must be developed to recover renewable resources, such as water, nutrients and also energy, from wastewater. Many studies have been conducted on membrane bioreactor containing microalgae for the purpose of biomass production [4]. In the current study Chlorella vulgaris was grown in membrane bioreactor to absorb nitrogen from wastewater and reduce pollution under mixotrophic condition in continuous mode.

\section{Experimental}

\section{Microalgae and experiment procedure}

A strain of C.vulgaris was prepared by Research Institute of Applied Science, Tehran, Iran. For this study COD was added as an organic carbon in all runs.

\section{Membrane bioreactor}

The experiments were conducted in submerged membrane bioreactor with working volume of 1.5 Liter. The reactor was purged with air, which introduced $\mathrm{CO}_{2}$ into the membrane bioreactor. Membrane bioreactor was illuminated by florescent lamp with light intensity of $2000 \mathrm{~lx}$ under light condition.

\section{Analyses}

Ammonium, Phosphate of liquid sample was measured based on standard methods [5]. Growth rate was determined by measuring optical density of samples by spectrometer (UNIC 2100) at $580 \mathrm{~nm}$ of wavelength.

\section{Results and Discussion}

As shown in Figure 1a, the microalgae biomass increased duration 23 days in continuous condition. The maximum microalgae concentration $(1.3 \mathrm{~g} / \mathrm{L})$ was achieved in membrane bioreactor in the $23^{\text {th }}$ day. The microalgae growth in membrane bioreactor was 2.3 times higher than batch mode. According to previous studies, light intensity is an important factor for mixotrophic cultivation affecting to microalgae growth [2]. The concentration of nitrogen in effluent is shown in Figure 1b. The microalgae were able to reduce the $\mathrm{N}-\mathrm{NH}_{3}$ concentration to $80 \%$ in membrane bioreactor that was higher than other heterotrophic studies [6]. Mixotrophic microalgae are ability to remove ammonium as previous study from wastewater and produce high biomass for biofuel production. 


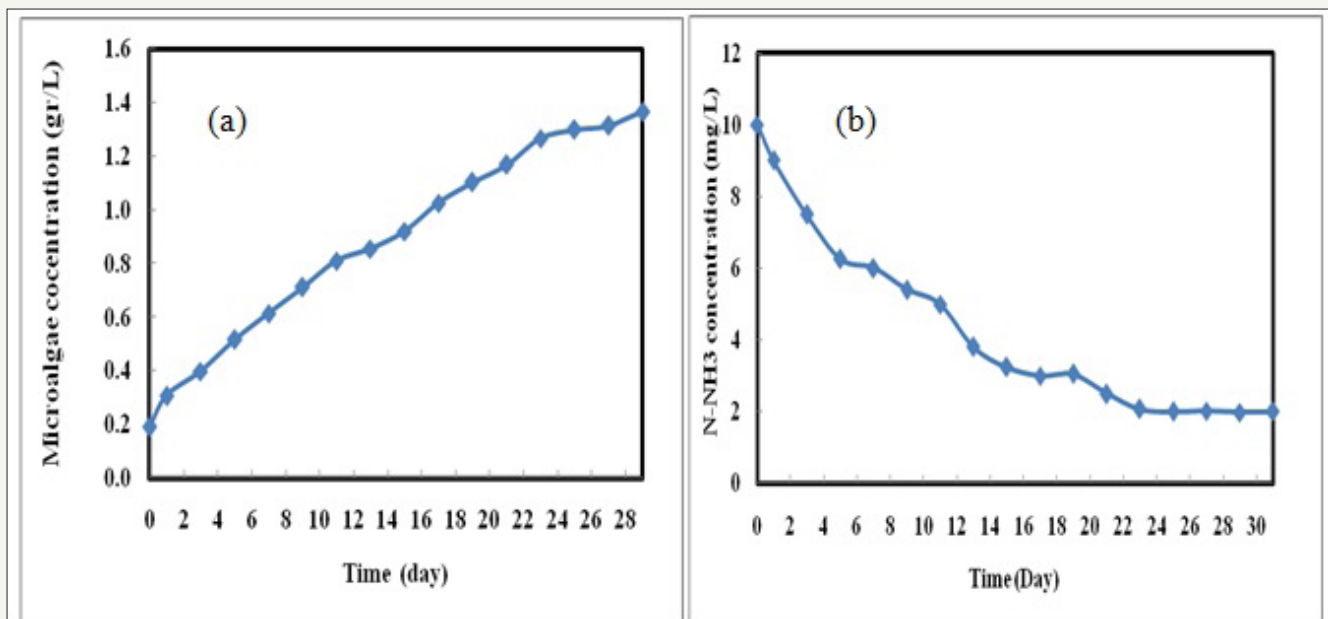

Figure 1 (a): biomass concentration in membrane bioreactor

Figure 1 (b): $\mathrm{N}_{-} \mathrm{NH}_{3}$ concentration of effluent in membrane bioreactor.

\section{Conclusion}

Based on this study, mixotrophic cultivation of microalgae have a good potential for the removal of nutrients from wastewater effluent. Nutrient removal in continuous mode were tested here. In this study, results show that the biomass production and ammonium consumption rate are increased by using membrane bioreactor. Removal of ammonium and biomass production was achieved by installing the submerged membrane as a solid liquid separator.

\section{References}

1. Chisti Y (2007) Biodiesel from microalgae. Biotechnol adv 25(3): 294306.

2. Ceron GMC, Miron AS, Fernandez SJM, Molina GE, Garcia CF (2006) Mixotrophic growth of the microalgae Phaeodactylum tricornutum influence of different nitrogen and organic carbon sources on productivity and biomass composition. Process Biochemistry 16(5): 689-694.

3. Kong W, Song H, Cao Y, Yang H, Hua S, Xia C (2011) The characteristics of biomass production, lipid accumulation and chlorophyll biosynthesis of Chlorella vulgaris under mixotrophic cultivation. Afr J Biotechnol 10(55): 11620-11630.

4. Singh G, Thomas P (2012) Nutrient removal from membrane bioreactor permeate using microalgae and in a microalgae membrane photoreactor. Bioresource Technology 117: 80-85.

5. APHA (2005) Standard methods for the examination of water and wastewater ( $21^{\text {st }}$ edn), American Public Health Association, NewYork, USA.

6. Babaei A, Mehrnia MR, Shayegan J, Sarrafzadeh MH (2015) The effect of dark and light cycle on nutrient removal by using membrane bioreactor containing microalgae. IWA Balkan Young Water Professionals conference, Greece.

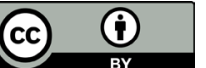

Creative Commons Attribution 4.0 International License

For possible submissions Click Here

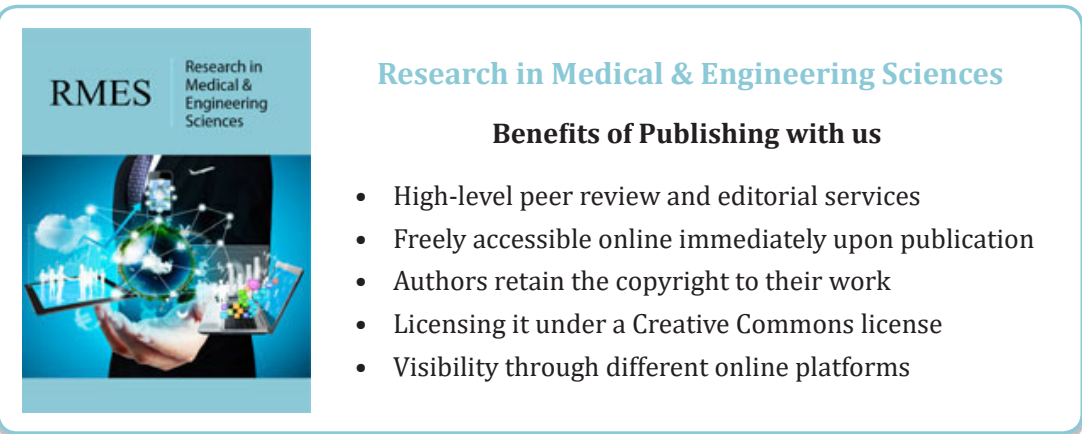

